\title{
Effects of lactic acid and lauricidin on the survival of Listeria monocytogenes, Salmonella enteritidis and Escherichia coli 0157:H7 in chicken breast stored at $4^{\circ} \mathrm{C}$
}

\begin{abstract}
Lauricidin and lactic acid were evaluated for their eVects on growth and survival of Listeria monocytogenes (L55), Salmonella enteritidis (S552) and Escherichia coli O157:H7 (E19) inoculated onto raw chicken breast. Fresh, raw chicken breasts were purchased immediately after slaughter and transported on ice to the laboratory within $20 \mathrm{~min}$. Each chicken breast was decontaminated by brieXy dipping in $70 \%$ ethanol and passed through a Xame of a Bunsen burner and then allowed to cool. The decontaminated Chicken breast was dipped in TSB broth, at room temperature $\left(25^{\circ} \mathrm{C}\right)$ for $15 \mathrm{~min}$, containing approximately log $9 \mathrm{CFU} / \mathrm{ml}$ of L. monocytogenes, S. enteritidis or E. coli O157:H7. Initial counts of L. monocytogenes, S. enteritidis or E. coli O157:H7 counts in chicken breast immediately after dipping in TSB broth were in the range of $\log 7-\log 8 \mathrm{CFU} / \mathrm{g}$. After inoculation, the chicken breasts were kept at room temperature for $20 \mathrm{~min}$ to allow attachment. Each inoculated chicken breast $\left(25{ }^{\circ} \mathrm{C}\right)$ was dipped in 0 (control - sterile water), $0.5 \%, 1 \%, 1.5 \%$ or $2 \%$ of lauricidin (w/v) or lactic acid (v/v) for 10,20 or 30min and then individually placed in oxygen-permeable polyethylene bags. Breasts were subjected to microbiological analyses after treatment (day 0) and after storage for 2, 5, 7, 10 and $14 \mathrm{~d}$ at $4{ }^{\circ} \mathrm{C}$. Initial counts of L. monocytogenes, S. enteritidis and E. coli O157:H7, in chicken breast treated with lauricidin decreased by 2.90, 1.31 and $2.27 \log \mathrm{CFU} / \mathrm{g}$, respectively. Lauricidin was more eVective in reducing $\mathrm{L}$. monocytogenes population than S. enteritidis and E. coli O157:H7 population. Dipping chicken breast in lauricidin for 30min caused a signiWcant reduction of L. monocytogenes, S. enteritidis and E. coli O157:H7 population compared to 10 and 20min dipping. Initial L. monocytogenes, S. enteritidis and E. coli O157:H7 counts on chicken breast treated with lactic acid decreased by 1.97, 1.71 and $2.59 \log C F U / g$, respectively. Lactic acid caused a higher reduction in initial S. enteritidis and E. coli O157:H7 counts compared to lauricidin.
\end{abstract}

Keyword: Poultry, Pathogen, Decontamination 